\title{
Correction to: Circulating blood levels of IL- 6 , IFN- $\gamma$, and IL-10 as potential diagnostic biomarkers in gastric cancer: a controlled study
}

Norma Sánchez-Zauco 1,2,3, Javier Torres ${ }^{4}$, Alejandro Gómez ${ }^{4}$, Margarita Camorlinga-Ponce ${ }^{4}$, Leopoldo Muñoz-Pérez ${ }^{4}$, Roberto Herrera-Goepfert ${ }^{5}$, Rafael Medrano-Guzmán' 6 Silvia Giono-Cerezo ${ }^{3}$ and Carmen Maldonado-Bernal ${ }^{* *}$

\section{Correction}

After publication of the original article [1] the authors found the following author names were incorrect and needed to be amended:

- In the original article, author Javier Torres' name was presented as J. Torres.

- In the original article author Roberto HerreraGoepfert's first name was incorrectly presented as Ramón.

Both these errors have been updated in the author list for this Correction. The original article has also been corrected.

\footnotetext{
Author details

'Laboratorio de Investigación en Inmunología y Proteómica, Hospital Infantil de México Federico Gómez, Dr. Márquez 162, Col. Doctores, 06720 Mexico City, Mexico. ${ }^{2}$ División de Auxiliares de Diagnóstico y Tratamiento UMAE Hospital de Especialidades, Centro Médico Nacional-Siglo XXI, IMSSI, Avenida Cuauhtémoc 330, Col Doctores, 06720 Mexico City, Mexico. ${ }^{3}$ Laboratorio de Bacteriología, Escuela Nacional de Ciencias Biológicas-IPN, Prolongación Manuel Carpio y Plan de Ayala, Santo Tomás, 11350 Mexico City, Mexico. ${ }^{4}$ Unidad de Investigación en Enfermedades Infecciosas, Hospital de Pediatría, Centro Médico Nacional Siglo XXI, IMSS, Avenida Cuauhtémoc 330, Col Doctores, 06720 Mexico City, Mexico. ${ }^{5}$ Departamento de Patología, Instituto Nacional de Cancerología, Secretaría de Salud, Av. San Fernando 22, Tlalpan, 1408 Mexico City, Mexico. ${ }^{6}$ Departamento de Sarcomas, Tracto Digestivo Bajo, UMAE Oncología, Centro Médico Nacional Siglo XXI, IMSS, Av. Cuauhtémoc 330, Col Doctores, 06720 Mexico City, Mexico.
}

Received: 25 September 2017 Accepted: 25 September 2017 Published online: 02 October 2017

\section{Reference}

1. Sánchez-Zauco N, Torres J, Gómez A, Camorlinga-Ponce M, Muñoz-Pérez L, Herrera-Goepfert R, Medrano-Guzmán R, Giono-Cerezo S, Maldonado-Bernal C. Circulating blood levels of IL-6, IFN- $\gamma$, and IL-10 as potential diagnostic biomarkers in gastric cancer: a controlled study. BMC Cancer. 2017;17:384. https://doi.org/10.1186/s12885-017-3310-9.

\footnotetext{
* Correspondence: cmaldobe@yahoo.com

${ }^{1}$ Laboratorio de Investigación en Inmunología y Proteómica, Hospital Infantil de México Federico Gómez, Dr. Márquez 162, Col. Doctores, 06720 Mexico City, Mexico

Full list of author information is available at the end of the article
}

(c) The Author(s). 2017 Open Access This article is distributed under the terms of the Creative Commons Attribution 4.0 International License (http://creativecommons.org/licenses/by/4.0/), which permits unrestricted use, distribution, and reproduction in any medium, provided you give appropriate credit to the original author(s) and the source, provide a link to the Creative Commons license, and indicate if changes were made. The Creative Commons Public Domain Dedication waiver (http://creativecommons.org/publicdomain/zero/1.0/) applies to the data made available in this article, unless otherwise stated. 